\title{
Armutsbekämpfung - eine vernachlässigte Aufgabe der Bundesregierung
}

Jahrzehntelang weigerte sich die Bundesregierung, Armut als gesellschaftliche Wirklichkeit und als Problem zu betrachten, das einer Lösung harrt. Aufgrund der „Vielschichtigkeit“ des Armutsbegriffs entziehe sich dieser einer allgemeingültigen Definition, argumentierte sie, und fügte hinzu, wegen des Rechtsanspruchs auf Leistungen der Sozialhilfe werde Armut hierzulande am Entstehen gehindert bzw. staatlicherseits wirksam bekämpft. ${ }^{1}$ Wohlfahrtsverbände, Gewerkschaften und Kirchen suchten das Kardinalproblem der sozialen Ungleichheit im vereinten Deutschland daher ,inoffiziell“ zu dokumentieren. Hiervon zeugen entsprechende Berichte der Caritas sowie des DGB, der Hans-Böckler-Stiftung und des Paritätischen Wohlfahrtsverbandes (ausführlicher dazu: Butterwegge 2012: 59 ff.). Auch manche Kommunen und einzelne Länder bauten früher als der Bund eine regelmäßige Berichterstattung zum Thema „Armut“ auf, wiewohl es den meisten schwer fiel, das Phänomen als solches offen zu benennen, weshalb die einschlägigen Materialsammlungen häufig verschämt ,Sozialberichte“ genannt wurden. Auf zentralstaatlicher Ebene lehnte die damalige CDU/CSU/FDP-Koalition wiederholt Anträge der Oppositionsfraktionen ab, denselben Versuch zu unternehmen. Erst die rot-grüne Koalition legte im April 2001, zweieinhalb Jahre nach dem mit sehr bald enttäuschten Hoffnungen verbundenen Regierungswechsel, einen Armuts- und Reichtumsbericht vor. Seither wird in unregelmäßigen Abständen, das heißt nicht unbedingt zur Mitte jeder Legislaturperiode - wie vom Bundestag seinerzeit beschlossen - regierungsoffiziell dokumentiert, welches Ausmaß die soziale Ungleichheit hierzulande erreicht hat (Butterwegge 2014: $322 \mathrm{ff}$.).

\section{Verharmlosung bzw. Verdrängung der sozialen Ungleichheit in den Armuts- und Reichtumsberichten}

Die bislang vier Armuts- und Reichtumsberichte der Bundesregierung weisen methodische Schwachstellen sowie inhaltliche Brüche und Widersprüche auf. Konzeptionell versuchte man zwar in Anknüpfung an den erreichten Forschungsstand,

1 BT-Drs. 10/6055: 10; BT-Drs. 13/3339: 2.

Zeitschrift für Politikwissenschaft 24. Jahrgang (2014) Heft 4, S. 493 - 501 
Armut qualitativ zu fassen und nicht auf fehlende finanzielle Ressourcen zu reduzieren, stellte den vorherrschenden Gerechtigkeitsbegriff jedoch aus Legitimationsgründen in Frage. Es komme, wurde behauptet, nicht so sehr auf die Umverteilung von materiellen Ressourcen, vielmehr auf die Bereitstellung von „Teilhabe- und Verwirklichungschancen" an. ${ }^{2}$ Man rekurrierte damit auf den Ansatz von Amartya Sen, einem indischen Ökonomen und Nobelpreisträger, der Armut zwar als Mangel an „Verwirklichungsmöglichkeiten“ begreift, ohne jedoch im Geringsten zu bestreiten, dass Letzterer mit einer Knappheit materieller Ressourcen bzw. monetärer Mittel (Einkommen und Vermögen) verbunden ist: „In einem reichen Land verhältnismäßig arm zu sein kann die Verwirklichungschancen selbst dann extrem einengen, wenn das absolute Einkommen gemessen am Weltstandard hoch ist." (Sen 2003: 112)

Die steigende Armutsquote wurde auf eine vor allem aus „externen Schocks“ wie den Terroranschlägen des 11. September 2001, dem Irakkrieg, dem Zusammenbruch des IT-Booms und spektakulären Bilanzskandalen von US-Unternehmen resultierende Wachstumsschwäche zurückgeführt. ${ }^{3}$ Schließlich fehlte die Einsicht, dass eine Regierungspolitik der Standortsicherung die soziale Polarisierung verschärft. Während Kapitaleigentümer, Begüterte und Spitzenverdiener als Gewinner dieser Form der ökonomischen Modernisierung immer reicher wurden, gehörten Millionen (Langzeit-)Erwerbslose, Geringverdiener/innen, Wohnungslose, Migrant(inn)en, (chronisch) Kranke, Behinderte, sozial benachteiligte Familien und Rentner/innen zu den Hauptverlierer(inne)n einer neoliberalen Reformpolitik, deren Höhe- bzw. Tiefpunkt das unter dem Kürzel „Hartz IV“ bekannte Vierte Gesetz für moderne Dienstleistungen am Arbeitsmarkt bildete (hierzu: Butterwegge 2015).

Auch für die im November 2005 gebildete zweite Große Koalition auf Bundesebene war Bildung der „Schlüssel für Teilhabe und Integration“, weshalb es im Dritten Armuts- und Reichtumsbericht heißt: „Schulische Bildung und berufliche Qualifikation sind die Grundlage für Teilhabe am Arbeitsmarkt und der beste Schutz gegen Arbeitslosigkeit und Einkommensarmut.“" Man stellte eine "Nationale Qualifizierungsinitiative“, die den Fachkräftebedarf der Wirtschaft decken helfen sollte, unter das Motto „Aufstieg durch Bildung“. Zwar kann ein Individuum durch die Beteiligung an (Aus-)Bildungsprozessen einer prekären Lebenslage entkommen, eine gesamtgesellschaftliche Lösung bietet sie allein freilich nicht. 
Armutsbekämpfung - eine vernachlässigte Aufgabe der Bundesregierung

Denn wenn alle Jugendlichen - nicht bloß jene mit einem Migrationshintergrund - besser gebildet wären, würden sie am Ende womöglich auf einem höheren intellektuellen Niveau immer noch um die zu wenigen Arbeits- und Ausbildungsplätze konkurrieren, weshalb es am Ende zwar mehr Taxifahrer mit Hochschulabschluss, aber nicht unbedingt weniger Armut gäbe.

Altersarmut stellte für die Bundesregierung weder im Dritten noch im Vierten Armuts- und Reichtumsbericht ein aktuelles Problem dar. Während das Stichwort „Kinderarmut" nur in den Fußnoten bzw. den dort aufgeführten Titeln zitierter Fachliteratur auftauchte, wurde Altersarmut als in ferner Zukunft drohende Gefahr heruntergespielt: „Die Einkommens- und Vermögenssituation der Älteren von heute ist überdurchschnittlich gut", hieß es im Vierten Armuts- und Reichtumsbericht. ${ }^{5}$ An gleicher Stelle wurde darauf hingewiesen, dass am 31. Dezember 2011 ,nur“ 436.210 Personen über 64 Jahren die Grundsicherung im Alter bezogen, was einem Anteilswert von rund 2,6 Prozent der Bevölkerung in dieser Altersgruppe entsprach, wohingegen der Anteil von Empfänger(inne)n von Mindestsicherungsleistungen aller Altersgruppen an der Gesamtbevölkerung bei 8,9 Prozent lag. Hierbei verkannte die Bundesregierung, dass die Dunkelziffer, das heißt der Anteil jener Menschen, die - ihnen eigentlich zustehende - Sozialleistungen wie die Grundsicherung nicht beantragen, weil sie zu stolz sind, weil sie sich schämen, weil sie den bürokratischen Aufwand scheuen oder weil sie fälschlicherweise den Unterhaltsrückgriff auf ihre Kinder und Enkel fürchten, gerade unter den Senior(inn)en besonders hoch ist. Man muss deshalb von weit mehr als einer Million Ruheständler(inne)n ausgehen, die auf oder unter dem Hartz-IV-Niveau (durchschnittlich 707 EUR pro Monat) leben. Mehr als 812.000 haben einen Minijob; etwa 128.000 davon sind 75 Jahre oder älter. Altersarmut ist eben kein bloßes Zukunftsproblem, sondern längst eine bedrückende Zeiterscheinung, wie jeder weiß, der alte Menschen frühmorgens Zeitungen austragen, öffentliche Toiletten putzen oder Regale im Supermarkt einräumen sieht.

Wer mit offenen Augen und ohne ideologische Scheuklappen durchs Land geht, kommt zu einem anderen Schluss als die Bundesregierung: Momentan verfestigt sich die Armut und frisst sich in die Mitte der Gesellschaft hinein. In manchen Ballungsgebieten und Boomtowns der Bundesrepublik drastisch steigende Mieten und Energiepreise gefährden sogar den Lebensstandard von Normalverdiener(inne)n und verstärken die Angst vieler Mittelschichtangehöriger vor dem sozialen Abstieg. Die soziale Ungleichheit wächst in erschreckendem Maße, ohne von den politisch Verantwortlichen als gesellschaftlicher Spaltpilz erkannt zu 
werden. Die soziale Polarisierung dürfte noch zunehmen und der gesellschaftliche Zusammenhalt schwinden, falls ein wirtschafts- und sozialpolitischer Kurswechsel ausbleibt. Aufgrund härterer Verteilungskämpfe um die knappen Finanzmittel des Staates ist jedoch keine Verbesserung des sozialen Klima zu erwarten.

\section{Reichtumsförderung statt Armutsbekämpfung}

Nach den gesellschaftlichen Ursachen der kaum mehr zu leugnenden Spreizung von Einkommen und Vermögen wird in keinem der bisherigen Armuts- und Reichtumsberichte gefragt. Allenfalls geraten die Auslöser persönlicher Notlagen wie Arbeitslosigkeit, Trennung bzw. Scheidung oder (Früh-)Invalidität ins Blickfeld der Berichterstatter. Umso wichtiger wäre die Beleuchtung struktureller Hintergründe, um persönlichen Schuldzuweisungen keinen Vorschub zu leisten sowie der Verantwortung von Staat, Wirtschaft und Gesellschaft gerecht werden zu können.

Unabhängig davon, ob die Regierungsberichte ihrer Aufgabe gerecht geworden sind, eine gute Datenbasis zur sozialen Ungleichheit in Deutschland und sinnvolle Empfehlungen für deren Verringerung zu liefern, hat sich die Bundesregierung damit verpflichtet, den gesellschaftlichen Zusammenhalt zu bewahren und sozialstrukturellen Verwerfungen zu begegnen. Tatsächlich könnten Armuts- und Reichtumsberichte die Basis für eine bessere Wirtschafts-, Sozial- und Finanzpolitik der Bundesregierung bilden, wenn sie die „Lebenslagen in Deutschland“ nüchtern analysieren, die Ursachen für wachsende Ungleichheit ergründen und entsprechende Handlungsempfehlungen geben würden.

Trotz aller Beschönigungs- und Beschwichtigungsversuche dokumentieren die Armuts- und Reichtumsberichte eine doppelte Spaltung: Erstens wachsen Armut und Reichtum gleichermaßen, sind also zwei Seiten derselben Medaille. Dies zeigt sich besonders deutlich beim Vermögen, das Arme gar nicht haben, weil es sich zunehmend bei wenigen Superreichen konzentriert, die über riesiges Kapitaleigentum verfügen und meistens auch große Erbschaften machen. Während die reichsten 10 Prozent der Bevölkerung fast 53 Prozent des Nettogesamtvermögens besitzen, kommt die ärmere Hälfte der Bevölkerung nur auf ein Prozent. Viele Millionen Menschen leben gewissermaßen von der Hand in den Mund, sind also nur eine Kündigung oder eine schwere Krankheit von der Armut entfernt. Bertolt Brecht hat es in einem Vierzeiler unnachahmlich prägnant ausgedrückt: „Armer Mann und reicher Mann / standen da und sah'n sich an. / Und der Arme sagte bleich: / Wär' ich nicht arm, wärst du nicht reich." Aufgrund dieses strukturellen Interdependenzverhältnisses kann Armut im Rahmen der bestehenden Gesell- 
Armutsbekämpfung - eine vernachlässigte Aufgabe der Bundesregierung

schaftsordnung auch nicht durch zunehmenden Reichtum beseitigt werden. Anders formuliert: Reichtumsförderung, wie sie die Bundesregierung trotz wechselnder Koalitionen seit Jahrzehnten betreibt, ist eben keine Armutsbekämpfung.

Zweitens geht der wachsende private Reichtum zwangsläufig mit einer öffentlichen Verarmung einher. Während sich das private Nettovermögen allein zwischen 2006 und 2011 um 1,5 Bio. Euro auf gut 10 Bio. Euro erhöht hat, ist das Nettovermögen des Staates in den letzten beiden Jahrzehnten um mehr als 800 Mrd. Euro gesunken. Geld ist also genug da, es befindet sich aber in den falschen Taschen, was den Staat auf Dauer handlungsunfähig macht, obwohl er die Aufgabe hat, sich derzeit verschärfende soziale Probleme zu lösen oder wenigstens zu lindern. Das in Art. 20 Abs. 1 Satz 1 GG und in Art. 28 Abs. 1 GG kodifizierte Sozialstaatsgebot unserer Verfassung verpflichtet die Regierung zur Armutsbekämpfung, aber auch zum Schutz der Bürger/innen vor Standardlebensrisiken wie Arbeitslosigkeit, schwerer Krankheit, Unfällen und Bedürftigkeit im Alter sowie zum sozialen Ausgleich (Gewährleistung eines Mindestmaßes an Bedarfs- statt Leistungsgerechtigkeit).

Da sich Banker, Broker und Börsianer, besonders rücksichtslose Spekulanten, die Taschen gefüllt haben, musste der Staat im Gefolge der Finanz-, Wirtschaftsund Währungskrise bluten, öffentliches Eigentum veräußern und mehr Schulden machen, um „Rettungspakete“ für Gläubigerbanken, Kapitalanleger und Großaktionäre schnüren zu können. Aufgrund des Merkel-Dogmas „Keine Steuererhöhungen!" bedeuten im Grundgesetz, in den Landesverfassungen und im europäischen Fiskalvertrag festgeschriebene „Schuldenbremsen“, also Kreditsperren, die nur wenige Ausnahmen zulassen, bei wachsender Armut und sozialer Unterversorgung größerer Bevölkerungsteile, dass der Wohlfahrtsstaat, wie man ihn bisher kannte, zugrunde gerichtet wird. Schon jetzt sind erhebliche regionale Disparitäten, unter denen das Ost-West-, das Nord-Süd- und das Stadt-Land-Wohlstandsgefälle besonders hervorstechen, unübersehbar. Wenn der Sozialabbau fortgesetzt wird, dürften die Städte der Bundesrepublik stärker zerfallen: in Luxusquartiere, wo sich die (Super-)Reichen hinter hohen Mauern verschanzen und von privaten Sicherheitsdiensten bewachen lassen, einerseits sowie in Elendsgettos, wo sich die Armen konzentrieren, andererseits.

Hier liegt zweifellos ein Hauptmangel aller bisherigen Regierungsberichterstattung: Nach den ökonomischen, politischen und sozialen Ursachen der kaum mehr zu leugnenden Spreizung von Einkommen und Vermögen wird in keinem Armuts- und Reichtumsbericht gefragt. Dass zunehmender Reichtum in einem Wirtschafts- und Gesellschaftssystem, welches auf dem Privateigentum an Produktionsmitteln, der Konkurrenz und der Mehrwertproduktion durch Ausbeutung 
menschlicher Arbeitskraft basiert, zwangsläufig Armut hervorbringt, blieb außer Betracht. Nur wer Armut und Reichtum als sich zwar individuell auswirkende, aber wechselseitig bedingende Erscheinungsformen einer wachsenden sozialen Ungleichheit erkennt und deren strukturelle Ursachen bekämpft, kann ihren den gesellschaftlichen Zusammenhalt und die Demokratie bedrohenden Auswüchsen wirksam begegnen.

Ebenso wie hinsichtlich der Armuts- und Reichtumsberichterstattung hat bisher noch jede Bundesregierung hinsichtlich der Armutsbekämpfung versagt. Dass man den Wohlhabenden offenbar nicht wehtun, sondern es Reichen und Superreichen selbst überlassen will, ob und wie sie sich für das Gemeinwohl engagieren, haben CDU, CSU und FDP im Vierten Armutsbericht zum Ausdruck gebracht. Sollte die Bundesregierung laut dem ursprünglichen Entwurf noch eruieren, ob und wie privater Reichtum über die Progression in der Einkommensteuer hinaus für die nachhaltige Finanzierung öffentlicher Aufgaben herangezogen werden könne, heißt es in der Endfassung: „Die Bundesregierung prüft, wie weiteres persönliches und finanzielles freiwilliges Engagement Vermögender in Deutschland für das Gemeinwohl eingeworben werden kann. "6 Offenbar favorisiert die Bundesregierung ehrenamtliche, Spenden- bzw. Stiftertätigkeiten sowie Mäzenatenund Sozialunternehmertum. Selbst wenn das Volk der Dichter und Denker zu einem Volk der Stifter und Schenker würde, wären die wachsenden sozialen Probleme der Bundesrepublik aber nicht zu lösen. Vielmehr wäre vor allem in der Steuerpolitik ein Umsteuern nötig: Erforderlich sind eine im obersten Bereich erheblich progressivere Einkommensteuer, die Rücknahme der steuerlichen Privilegierung von Kapitalerträgen durch die Abgeltungssteuer in Höhe von 25 Prozent, die Wiedererhebung der 1997 ausgesetzten Vermögensteuer und eine Erbschaftsteuer, die auch große Betriebsvermögen erfasst. Schließlich ist es keine Leistung, der Sohn oder die Tochter eines Konzerngründers zu sein!

\section{Die geistige Armut der Großkoalitionäre}

CDU, CSU und SPD (2013) zeigen für das Kardinalproblem unserer Gesellschaft keine Sensibilität. Vielmehr kommt das Wort „Reichtum“ im Koalitionsvertrag für die 18. Legislaturperiode auf 185 Seiten nur als „Ideenreichtum“ bzw. als „Naturreichtum“ und der Begriff „Vermögen“ nur als „Durchhaltevermögen“ bzw. im Zusammenhang mit der Vermögensabschöpfung bei Kriminellen vor. 
Armutsbekämpfung - eine vernachlässigte Aufgabe der Bundesregierung

„Armut" taucht zwar zehn Mal im Koalitionsvertrag auf, größtenteils aber in fragwürdiger Art und Weise.

Zuerst firmiert das Motto „Altersarmut verhindern - Lebensleistung würdigen“ (10) als Zwischenüberschrift zur Rentenpolitik der künftigen Regierungskoalition. Darunter heißt es, die sozialen Sicherungssysteme, auf die sich die Menschen in unserem Land verlassen können müssten, schützten vor Armut und seien Ausdruck des Zusammenhalts unserer Gesellschaft. Beide Formulierungen legen den Schluss nahe, dass Altersarmut in Deutschland (noch) nicht existiert, denn von der Notwendigkeit ihrer Bekämpfung, Verringerung oder Beseitigung ist nirgends die Rede.

CDU, CSU und SPD wollen „,den Kampf gegen Bildungsarmut fortsetzen und intensivieren“ (33). Während von Kinder- und Jugendarmut an keiner Stelle die Rede ist, erscheint der Begriff „Bildungsarmut“", mit dem im Koalitionsvertrag die Alphabetisierungsbemühungen von Bund und Ländern begründet werden, insofern missverständlich, als er zur Verwechslung von Ursache und Wirkung geradezu einlädt. Trotz verbreiteter Vorurteile sind Menschen nicht wegen mangelnder Bildung arm - drei Viertel der Beschäftigten im Niedriglohnsektor verfügen über einen Berufs-, 11 Prozent sogar über einen Hochschulabschluss -, sondern Armut führt zu ihrer Benachteiligung im Bildungsbereich. Ein schlechter oder fehlender Schulabschluss verringert zwar die Erwerbschancen, wirkt sich aber kaum nachteilig auf den Wohlstand einer Person aus, wenn diese vermögend ist oder Kapital besitzt.

Dem hierzulande vorherrschenden Armutsverständnis gemäß wird das Phänomen im Koalitionsvertrag von CDU, CSU und SPD hauptsächlich mit der sogenannten Dritten Welt in Verbindung gebracht. Nicht weniger als vier Mal taucht Armut in diesem Zusammenhang auf, der jedoch verdeckt, dass sie in einem reichen Land wie der Bundesrepublik - wenn auch in anderer, weniger dramatisch wirkender Form - gleichfalls existiert und für die davon Betroffenen hier sogar erniedrigender, demütigender und demoralisierender sein kann. Forschung und Forschungskooperation (besonders mit Afrika) werden als Instrumente genannt, mit denen der "Teufelskreis von Armut und Krankheit in Entwicklungsländern“ durchbrochen werden könne (34). Folgerichtig setzen sich die Regierungsparteien für „nachhaltige Entwicklung und Armutsbekämpfung“ (168) ebendort ein. Ziel der großkoalitionären Entwicklungspolitik sei es, ,auf der Grundlage unserer Werte und Interessen [??] weltweit Hunger und Armut zu überwinden.“ (180) In den ärmsten Ländern der Erde sollen die Anstrengungen zur „Überwindung von Hunger und Armut"“ (182) verstärkt werden. 
Gleich drei Mal wird im Koalitionsvertrag das Wort „Armutswanderung“ bzw. „Armutsmigration“ (108) verwendet. Gemeint sind Bulgaren und Rumänen, denen man eine ,ungerechtfertigte Inanspruchnahme von Sozialleistungen“ vorwirft, wodurch deutsche Kommunen übermäßig belastet würden. Auch wenn die sozialen Problemlagen einzelner Großstädte, etwa Duisburg und Dortmund, bei der Unterbringung, Existenzsicherung, Gesundheitsversorgung und Integration treffend beschrieben werden, leistet der Koalitionsvertrag rassistischen Ressentiments dadurch Vorschub, dass er der „Migration in die sozialen Sicherungssysteme" entgegentritt, ohne Not und Elend der Herkunftsländer zu erwähnen und zu berücksichtigen, dass die Mehrheit der zugewanderten EU-Bürger teilweise hoch qualifizierte Arbeitskräfte in sozialversicherungspflichtigen Beschäftigungsverhältnissen sind.

Glaubt man dem Koalitionsvertrag von CDU, CSU und SPD, gibt es in Deutschland gar keine Armut, es sei denn, dass sie durch unerwünschte Zuwanderer importiert wird. Mit dem im Koalitionsvertrag auch von der Sozialdemokratie bestätigten Merkel-Mantra „Keine Steuererhöhungen - für niemand!“ wird die selbst im Vierten Armuts- und Reichtumsbericht der schwarz-gelben Koalition eingeräumte Verteilungsschieflage akzeptiert und das Auseinanderfallen der Gesellschaft forciert. Selbst der allgemeine Mindestlohn, den die Koalitionspartner ab 1. Januar 2015 per Gesetz eingeführt haben, steht für sie nicht im Kontext der Armutsbekämpfung, obwohl der ausufernde Niedriglohnsektor das Haupteinfallstor für heutige Erwerbs- und künftige Altersarmut bildet. Sonst hätten CDU, CSU und SPD die offizielle Lohnuntergrenze nämlich kaum bis 2017 bei 8,50 Euro festgeschrieben. Denn schon heute reicht diese Höhe selbst bei Vollzeiterwerbstätigkeit nicht aus, um in Würde leben, eine Familie ernähren und ohne Inanspruchnahme der Grundsicherung altern zu können.

\section{Literatur}

Butterwegge, Christoph, 2012: Armut in einem reichen Land. Wie das Problem verharmlost und verdrängt wird, Frankfurt a. M./New York (3. Aufl.).

Butterwegge, Christoph, 2014: Krise und Zukunft des Sozialstaates, Wiesbaden (5. Aufl.).

Butterwegge, Christoph, 2015: Hartz IV und die Folgen. Auf dem Weg in eine andere Republik?, Weinheim/Basel (2. Aufl.).

CDU/CSU/SPD, 2013: Deutschlands Zukunft gestalten. Koalitionsvertrag zwischen CDU, CSU und SPD, 18. Legislaturperiode (https://www.cdu.de/sites/ default/files/media/dokumente/koalitionsvertrag.pdf; Stand: 12.1.2015). 
Sen, Amartya, 2003: Ökonomie für den Menschen. Wege zu Gerechtigkeit und Solidarität in der Marktwirtschaft, München (2. Aufl.).

Korrespondenzanschrift:

Prof. Dr. Christoph Butterwegge

Universität zu Köln

Humanwissenschaftliche Fakultät

Institut für Vergleichende Bildungsforschung und Sozialwissenschaften

- Politikwissenschaft -

Gronewaldstr. 2

50931 Köln

E-Mail: hf-politikwissenschaft@uni-koeln.de 